\title{
Multi-Sensor Approach for Monitoring Pipelines
}

\author{
Salihu O. Aliyu, Innocent O. Agbo, Saidu Muslim and Elizabeth N. Onwuka \\ Department of Telecommunication Engineering, Federal University of Technology, Minna, Niger State, 234, \\ Nigeria.
}

Received: 04 January 2017; Accepted: 13 February 2017; Published: 08 November 2017

\begin{abstract}
Pipeline vandalization is one of Nigeria's economy killer, since Nigeria economy to a great extent relies on oil in that capacity. Therefore, a third party damage to petroleum pipelines can be cataclysmic if undetected and prevented. This act results in budgetary misfortunes, ecological contamination and incessant death and loss of properties worth millions as an aftereffect of vandalization. Consequently, it is very paramount to protect these pipelines from vandals through intelligent monitoring systems. Several efforts have been made towards providing a reliable monitoring system for oil pipeline, however, no practically implementable solution have been achieved. Therefore, a pipeline monitoring system using multi-sensors is presented herewith. The sensor array consists of a Passive Infrared (PIR), vibration and sound sensor. An uninvolved infrared (PIR) sensor was utilized to detect intruders before they get in contact with the pipeline and for affirmation of intruders, sound and vibration sensor were set up. As the PIR recognizes an on-coming human the sound and vibration sensors affirms if the human is an intruder(s). An intrusion message containing the location of the vandals is sent to the appropriate authority by the microcontroller via a connected GSM module. Results obtained proved the system as a viable solution for detecting pipeline vandals.
\end{abstract}

Index Terms: Vandalization, Petroleum pipeline, PIR sensor, sound sensor, vibration sensor, GSM module, GPS module.

(C) 2017 Published by MECS Publisher. Selection and/or peer review under responsibility of the Research Association of Modern Education and Computer Science.

\section{Introduction}

Nigeria is the biggest oil producer in Africa and the eleventh biggest in the world. The backbone of Nigeria's economy is the Petroleum sector, contributing around $90 \%$ of the country's remote trade income and around $25 \%$ of the Gross Domestic Products (GDP). A huge extent of the Nation's oil is delivered coastal and

* Corresponding author. Tel.:

E-mail address: salihu.aliyu@futminna.edu.ng 
transported by pipelines [1]. Pipeline framework as a medium of transportation is normally credited to exceptionally delicate items such as unrefined petroleum, common gas and mechanical chemicals, in which unattended issues in their operation results in inconceivable calamity. These issues incorporate terrorism assaults, vandalism and robbery of the pipeline content. The requirement for executing satisfactory security frameworks for pipeline is of incredible substance, however first keeping in mind that discussion about pipeline vandalism itself and the requirement for a gadget that can stop the demonstration of vandalism is paramount. Pipelines have been utilized to adequately transport fundamental wares required for human presence over a long separation. Examples of such commodities include water, regular gas, petroleum product and liquid hydrocarbons. Pipeline failure is most times brought on by erosion, wear, deliberate and accidental harm among others. Such failure can prompt loss of life and property. As of January 2016 Nigeria was at that time losing 470 million Naira per day to pipeline vandalism [1]. In 1998 and 2009, Nigeria lost more than two thousand individuals because of pipeline explosion across the nation; most explosions are created by inhabitants scooping fuel from vandalized pipelines. A typical scenario of oil scooping is as shown in Fig. 1.

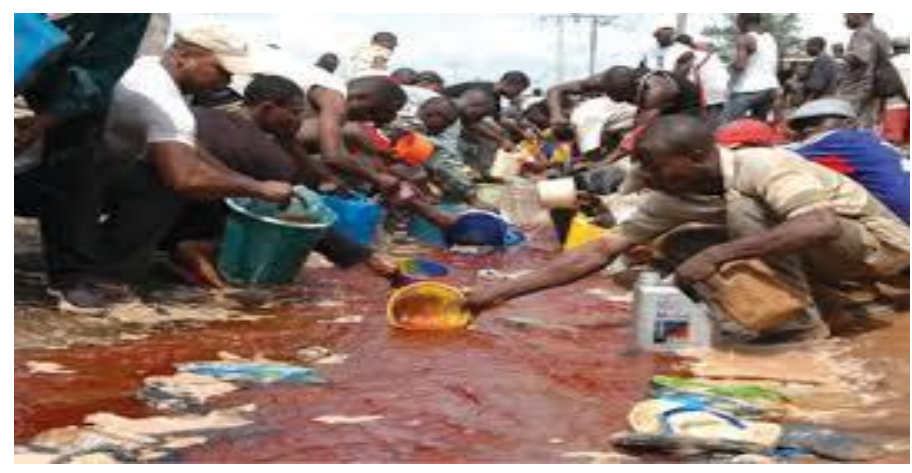

Fig.1. Scene of People Scooping Petroleum Product

Considering the damages associated with oil pipeline vandalization, it is of utmost importance to prevent these losses, thus, a methodology is proposed herewith to mitigate pipeline vandalization using multi-sensors. The rest of this paper is organized as follows: section 2 presents review of related works, while materials and methodology used is discussed in section 3. Section 4 presents testing of the developed prototype and discussion of results, while conclusion and recommendation for future work is presented in section 5 .

\section{Related Works}

Endeavors to monitor and avoid pipeline vandalism did not begin today. Different researchers have utilized distinctive methodologies and innovations to impede uncontrollable endeavors to access oil substance being transported by means of pipelines. In this section, a review of previous works that endeavored to design and implement comparable work like the proposed work herewith is presented.

The authors in [2] presented a microcontroller based alarm system for detection of pipeline vandals. The framework is a configuration to identify leakage whenever a rubber on the pipe is removed. This action triggers the microcontroller which quickly alert concern authorities for necessary action. The quality of this design lies in the ability of closing down liquid streams. Furthermore it is all around hid in a manner that the vandals can't, without much of a stretch, wreck it. It shortcoming is that the optical filaments are laid along the pipeline making it costly.

The work done by Obodoeze, 2012, [3] has its own design and development of the system in both hardware and software requirements. The hardware subsystems include the hardware modules used to realize the physical test-bed for the oil pipeline vandalism surveillance and monitoring system. The software sub-systems 
include the software modules used to realize the sensor initializations and communication, data transfers from the deployed test-bed to an online ftp server, designated email addresses, and mobile phone numbers. The system was design to enable data visualization from the ftp server to web-based SCADA server, thus, authorized personnel can view videos/photos captured from a vandalization scene at the oil pipeline infrastructure. The quality of the design lies with the way pictures are taken and sent to the email of the beneficiary in addition to a remote server. Its shortcoming is that the framework utilizes sun powered board to control the whole gadget and the camera, accordingly when the sun based board is found it gives the vandals a thought that security system may be installed, consequently the gadget is crushed before vandalism happens.

Another system proposed in [4] as a component of this exploration, is a framework that was designed around trans-beneficiary, microcontroller, low level computing construct program, GSM module for correspondence, caution and power supply. The framework was designed such that whatever point a spillage is identified in the pipeline, it will trigger caution and send SMS to the control unit. The control unit utilizes the framework interface to determine the definite purpose of the spillage identified for conceivable activity. The shortcoming of the framework is that if there should be an occurrence of vandalism the framework ringer tells the vandals its careful position and all things considered gives the vandals an edge over the framework.

In [5], their model embraced a light-to-power detecting strategy to identify spill in pipeline. Utilizing light recognition through an opening in a channel to show the event of a break, the authors used a fitting transducer (i.e. a light-to-power sensor) which for their situation was a Light Dependent Resistor (LDR). In this way, by interfacing the LDR in a resistive divider arrangement, their goal was accomplished. The quality of this work lies in its capacity to give definite area of the vandals which is sent to the control room for appropriate investigation, while its shortcoming is that it relies upon light to identify possible vandals, consequently, it can't distinguish vandals during evening time.

An approach introduced in [6][7] involve the use of leak detection based on transient modeling, the leak is modeled as a pressure- and density-dependent function, which helps to improve the leak detection capability during transient flow such as pipeline shut down and start up. The upstream and downstream estimations of these stream parameters stay unaltered before the occurrence of a hole. At the point when there is a release, a sign which go at the speed of sound along the pipeline portions is transmitted to both closures of the pipeline. These impacts, for example, change in the estimation of weight; stream rate and temperature are given by the weight sensors, flowmeters and temperature sensors separately. As a consequence of this, the varieties in this thermodynamic streaming component perceive the appearance of break, detect it and decide its amount. Quality of the outline is that transient displaying for pipeline made it tremendous for break to be analyzed and evaluated for an extensive assortment of pipeline design. Its shortcoming is that it can't deal with complex pipeline system or much information handling for single pipeline. In [8],[9], a methodology which comprises of handset (GSM module), microcontroller, power supply and alert units was proposed. Reenactment of the different units was done independently utilizing visual displaying application, Proteus 8 Professional. The majority of the parts utilized as part of the exploration were accessible on the product and other choices with comparative particulars were utilized for segments that were not accessible on the Proteus for reenactment purposes. A nonstop electrical way was given by safe sensor and any break in the sign way causes end of sign and gives discernible change in the condition of the framework. The framework was tried on execution of the undertaking of identifying breakage on pipelines and the accurate area of such issues. The significant quality of the framework lies in its capacity to send SMS to the best possible channels. The shortcoming of the framework is that only when an impact on the pipe is detected by the sensor that a signal is sent.

A framework which utilizes WSN was proposed in [10]-[12]. A remote sensor system, is an accumulation of sensor hubs interconnected by remote correspondence channels. Every sensor hub is a little gadget that can gather information from its encompassing territory, complete basic calculations, and speak with different hubs or with the Base Station (BS). Such systems have been acknowledged because of late advances in small scale electromechanical frameworks and are relied upon to be generally utilized for applications, such as, environment observing, home security, modern procedure checking, and medicinal services applications. The quality of WSN is that every sensor hub is a little gadget that can gather information from its encompassing 
territory, complete basic calculations, and communicate with different hubs or with the base station (BS). It shortcoming accompanies issues including absence of productive steering conventions, system security and connection quality.

A framework that utilizations robot was exhibited in [13]; it checks if the color of the surface of the object read by the colored sensor is black (indicating a hole) as defined in the program. If so, then the robot stops for 5 seconds while the warning sound plays which act as an alarm. It increases it counter by 1 on every detection and the total number of cracks detected is displayed after the last detection. The distance covered is also calculated using the motor function and this is also displayed on the NXT (Next generation from previous design) display. The check is carried out within a specified time in the code, to maintain a strategic distance from intemperate utilization of the processor in the sensor readings and a boundless circle. Also, it checks for a break which was demonstrated with a white small shade of $0.2 \mathrm{~cm}$ wide with the assistance of it shading sensor. If the white color is detected, the robot pauses for 2 seconds and makes a warning sound as an alert. It also increases a separate counter for this and calculates the distance covered and this information is displayed on the NXT display as indicated in the program. Quality of the robot is that the robot is fit for identifying breaks that are more evident to the eye. Its shortcoming is that it is too huge and can be spotted effortlessly by the vandals.

The basic rule of operation of broadly used pipeline checking frameworks, for example, the Distributed Acoustic Sensors (DAS) is revolved around discovery of sound minor departure from the pipeline [14]. In spite of the innovation utilized as part of the discovery and the technique for sign handling utilized, the major amount being distinguished and read is sound. Their work was based on this crucial idea yet was developed utilizing microcontrollers and DTMF innovation. Shortcoming of the framework is that only when the vandals strike the pipeline before the framework distinguishes.

Power has been a serious issue with pipeline monitoring system. Such in [15], a pipeline monitoring system based on piezoelectric sensors was proposed to replace the existing high-power-consuming sensing system. The system consists of wireless impedance nodes that are operated with energy-aware software techniques. The experiments conducted in a real pipeline test bed show that the proposed system, indeed, detects pipeline structural deformation and also manages the monitoring system continuously with a guaranteed operation time. This system also operates on wireless sensor network (WSN) which has a problem with efficiency with regards to routing protocols, network security and link quality.

The authors in [16] developed a new fault-tolerant sensor network architecture for monitoring pipeline infrastructures. The proposed architecture is an integrated wired and wireless network. The wired part of the network is considered the primary network while the wireless part is used as a backup among sensor nodes when there is any failure in the wired network. This architecture solves the current reliability issues of wired networks for pipelines monitoring and control. But such project is financially not easy to handle.

An approach to minimizes energy consumption using dynamic duty cycle was proposed in [17]. Dynamic Duty Cycle is the percentage of radio-active period over the entire cycle period (i.e. active and inactive period together). Generally, periodically putting the sensor nodes into sleep state when there is no network activity can effectively save energy in WSN because unnecessary idle listening wastes a great amount of power. The primary goal of duty cycling approaches is to put nodes to low duty cycle operation for as long as possible. In the pipeline monitoring applications, the monitoring sensor nodes are often inaccessible after deployment and hence are expected to have very long life time. Duty cycling schemes are considered the highly efficient methods to reduce the energy consumed by radio transceivers. However, keeping a short period of time for idle listening is a challenging task because other network performance measures such as network throughput and connectivity should not be compromised.

In [18], the authors investigated and proposed an oil and gas pipeline monitoring solution based on wireless sensor networks with reliability focus on leakage sensing and wireless data communication. The system is developed indigenously and provides capability of reporting pipeline health related statistics stretched over large geographical areas.

Artificial Neural Network is a technique of artificial intelligence that has the ability to learn from experience, improving its performance and adapt to changes in the environment. The work in [19] is based on acoustic 
method and on-line prediction of leak location using neural artificial networks. Audible noises generated by leakage were captured by a microphone installed in a $60 \mathrm{~m}$ long pipeline. The sound noises were decomposed into sounds of different frequencies: $1 \mathrm{kHz}, 5 \mathrm{kHz}$ and $9 \mathrm{kHz}$. The dynamics of these noises in time were used as input to the neural model in order to determine the occurrence, magnitude and location of a leak (outputs of the model). The results have shown the great potential of the technique and of the developed neural models. For all on-line tests, the neural model 1 (responsible for determining the occurrence and magnitude of the leak) showed $100 \%$ accuracy, except when the leakage occurred through a small orifice $(1 \mathrm{~mm})$, with leak located at $3 \mathrm{~m}$ from the microphone. In all cases where neural model 1 detected the leak, the neural model 2 (responsible for determining the location) could accurately predict the exact location of the leak, except for an orifice of 3 $\mathrm{mm}$, with leakage occurring at the inlet end of the pipeline, showing an error of approximately $1.2 \mathrm{~m}$. The main advantages of neural networks are: the possibility of efficient manipulation of large amounts of data and its ability for generalization.

The authors in [20] presented a novel observing and pre-cautioning framework in light of multi-seismic sensors. Numerous sensors and handling modules which are set close by the pipeline gather the seismic signs created by various targets. Also in [21], The authors basic idea of the model is that it combines the fiber sensing technology to collect soil vibration around pipelines, online signal processing technology and artificial neural network for recognition, leading to an intelligent signal processing system. A recognition rate of $95.3 \%$ was obtained from the proposed setup.

$\mathrm{Yu}$ and Guo, 2012, [22] used an algorithm which is able to enhance the capabilities of gathering, managing, analyzing of pipeline state information, and further improving the pipeline efficiency. The algorithm made full use of the advantages of multi-hop, data fusion and energy consume balance technologies, which provided approving network performance on lifetime, data packet delay, and assured that urgency data packet was uploaded in time.

Unlike previous works, an approach which uses multiple sensors to monitor pipelines and has the ability to provide early notification of possible vandals is presented in this paper. The method employed and materials used are discussed as follows.

\section{Materials and Methods}

The methodology proposed here includes the utilization of GPS system to detect pipeline location, a GSM module to send notification. The sensors used to monitor the pipeline are motion, sound and vibration sensors unlike the work in [21] which made use of vibration sensor only. The motion sensor is in charge of the first assignment of this system which is to detect human movement 10-15 meters away from the pipeline, this is trailed by the sound sensor which detects the sound of the pipeline when it is stroked or the sound from the vandals' use of machinery. The vibration sensor and the sound sensor becomes possibly the most important factor when the demonstration of vandalism begins on the pipeline itself. The vibration within and around the pipe and sound made between the vandal's device and the pipeline is identified by these sensors. All sensors send their reaction to the microcontroller which conveys these by means of the GSM Module. In addition, the coordinate of the vandalization is identified with the guide of a GPS module and sent through GSM system to the appropriate authorities. The design consist of an ATMEGA8 microcontroller device, PIR motion sensor, vibration sensor, sound sensor, LCD, GPS, GSM MODEM, 74HC4052 serial port multiplexer and power supply, as shown in Fig 2. 


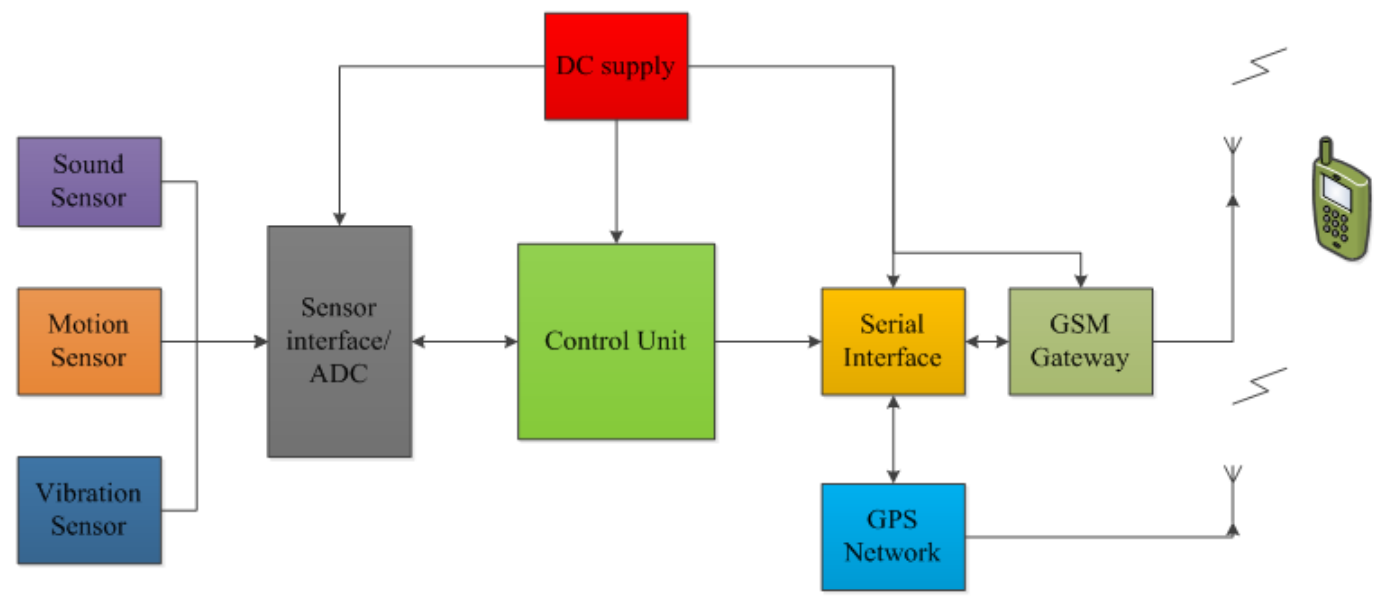

Fig.2. System Functional Block Diagram

The system design consist of the hardware components and a program which runs on the microcontroller chip which serves as the brain of the system.

\subsection{Hardware Design and Considerations}

The hardware consists of the following units: Control unit, Sensors, Power unit, Display and Communication unit. Each of this is discussed thus. The control unit was built around an ATmega8 microcontroller which is a CMOS Reduced Instruction Set Computer (RISC). It is an 8-Bit Microcontroller, In-system Programmable with Flash code storage, re-programmable up to 1000 write/rewrite times. Its Features includes 32 working registers, single clock cycle execution giving up to $1 \mathrm{MIPs} / \mathrm{MHz}$, 6-channel ADC with 10-bit Accuracy, 23 Programmable I/O Lines, 4.5 to $5.5 \mathrm{~V}$ operating voltage. Its in-built features motivated our choice as compare to the $8051 / 8052$ family which requires external ADC chip, and crystal oscillator, amongst others. The microcontroller is the main component of the control unit and interfaces with other units of the system as shown in Fig. 3.

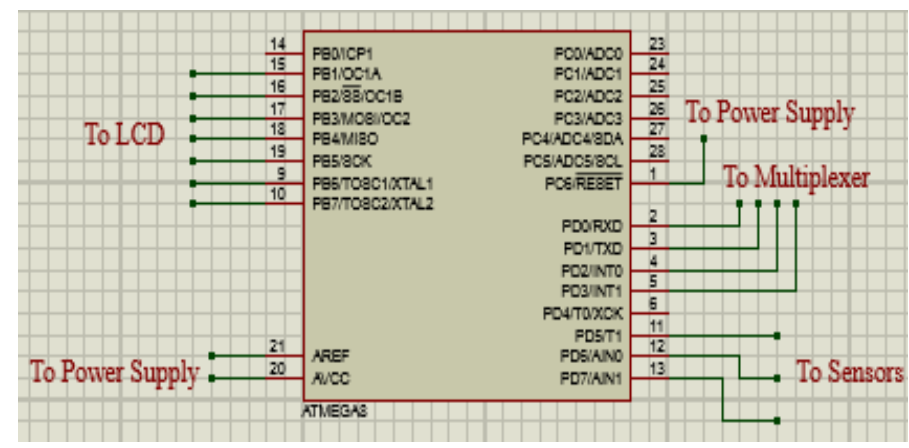

Fig.3. Circuit Showing the Control Unit

Three different sensors were used to monitor the pipeline and surroundings: motion, sound, and vibration sensors. The motion sensor (or motion detector) monitors the pipeline surroundings to detect the presence of possible vandals. It uses one or multiple technologies to detect movement in an area. Since the target vandals in 
this work are human, a PIR sensor was selected due to its ability to detect or differentiate humans from nonhumans. The PIR sensor is depicted in Fig. 4.

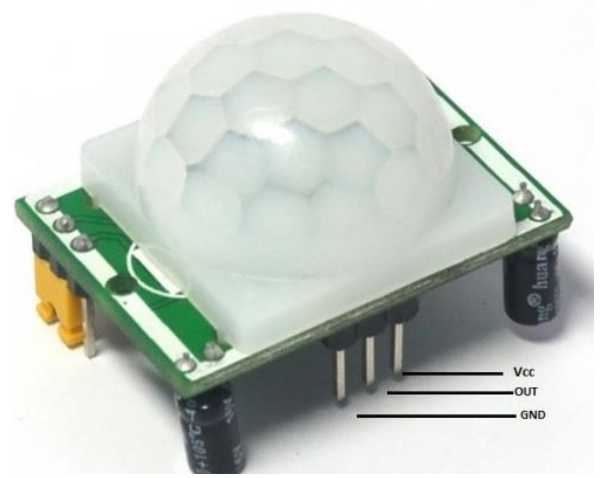

Fig.4. Passive Infrared (PIR) Sensor Module

In most cases, destroying pipelines involves hitting it with some kind of specialized instruments which results in the pipe making loud sound and possible vibration from the pipe and surrounding soil. Therefore, the sound sensor monitors the surrounding sound to know if it is related to pipeline vandalization. There is need to know the distance travelled by sound in the pipe using the speed of sound. This is needed to know the coverage of the proposed system along the pipeline and to estimate the next point of installation of the device. The speed of sound is the distance travelled per unit time by a sound wave propagating through an elastic medium. In dry air at $20^{\circ} \mathrm{C}$, the speed of sound is $343.2 \mathrm{~m} / \mathrm{s}(1,126 \mathrm{ft}$. $/ \mathrm{s})$, which is equivalent to $1,236 \mathrm{~km} / \mathrm{h}$ (i.e. a kilometre in $2.914 \mathrm{~s})$. It travels about 4.3 times as fast in water $(1,484 \mathrm{~m} / \mathrm{s})$, and nearly 15 times as fast in iron $(5,120 \mathrm{~m} / \mathrm{s})$, as in air at $20{ }^{\circ} \mathrm{C}$. Equation (1) can be used to calculate the speed of sound in pipes either in rigid or soft pipe as the case may be.

$$
\text { speed }=\frac{1}{\sqrt{\rho\left(\frac{1}{B}+\frac{D}{E \times e}\right)}}
$$

Where $\rho$ is Density $(\mathrm{kg} / \mathrm{m} 3)$, B is Bulk modulus (Pa), D is Inner diameter (m), e is Pipe thickness (m), and E is Young's modulus $(\mathrm{Pa})$. Once the speed is determine, the distance travelled by the sound through the pipe can be computed for a given time interval. The sound sensor module is as shown in Fig. 5.

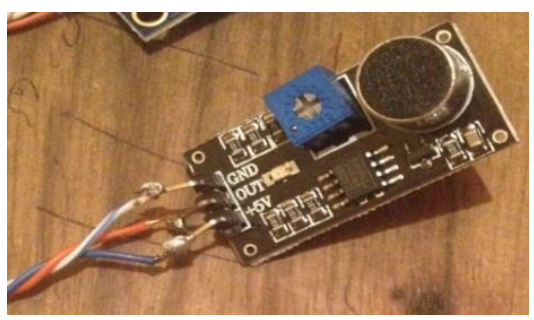

Fig.5. Sound Sensor Module

In addition to sound, vibration also accompanies a strike on the pipeline. To capture this, a vibration sensor 
was placed on the pipe. A typical vibration sensor incorporate a piezoelectric crystal and converts vibrations into an equivalent electrical signal such as a resistance or voltage. The vibration sensor used is shown in Fig. 6 .

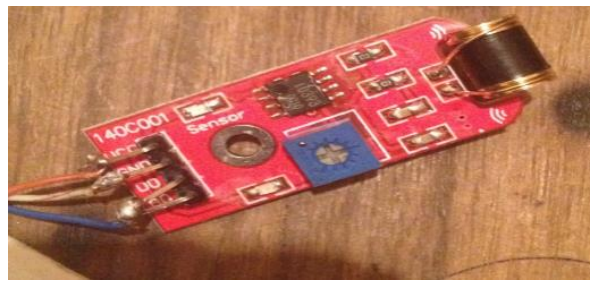

Fig.6. Vibration Sensor Module

\subsection{Communication Link}

This unit comprises of the GPS and the GSM module and helps to get the coordinates of the ongoing vandalism, then communicates it through GSM network to concern authorities. Thus a GPS and GSM modules were used to achieve this stage.

The Global Positioning System (GPS) is a space-based navigation system that provides location and time information in all weather conditions, anywhere on or near the Earth where there is an unobstructed line of sight to four or more GPS satellites. The system provides critical capabilities to military, civil, and commercial users around the world. The GPS module is shown in Fig. 7.

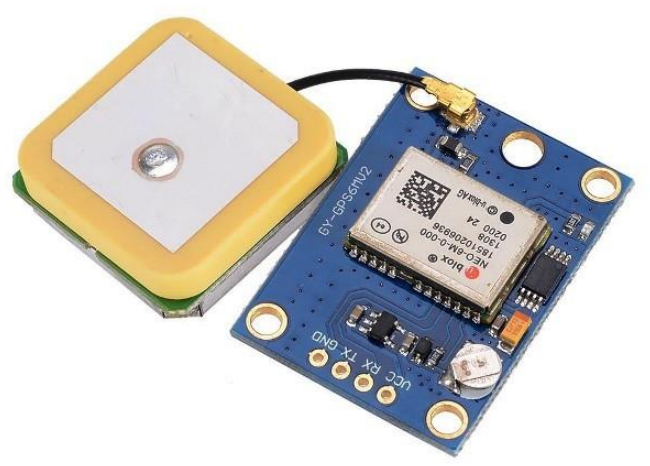

Fig.7. GPS Sensor Module

In the case of vandalism detection, the microcontroller reads the coordinate of the location and sends a notification message using the available GSM network. The major Nigeria network providers (MTN, Airtel, Etisalat, and Globacom) have been used as a means of communication between the pipeline monitoring system and security personnel. The SIM800 GSM module was used to communicate between the system and security personnel. These modules uses the RS232 standard serial communication protocol and since the microcontroller used can only interfaced with a single serial device, there is need to use a serial port multiplexer.

\subsection{Serial Port Multiplexer (74HC4052)}

The microcontroller used has one serial port and since we are imploring the use of two devices that uses serial communication, as such the 74HC4052 multiplexer is implored. The 74HCT4052 is a dual single-pole 
quad-throw analogue switch (2x SP4T) suitable for use in analogue or digital 4:1 multiplexer/de-multiplexer applications. Each switch features four independent inputs/outputs (nY0, nY1, nY2 and nY3) and a common input/output (nZ). A digital enable input (INH) and two digital select inputs (A and B) are common to both switches. When INH is HIGH, the switches are turned off. The function table of the 74HC4052 is shown in Table 1, while the circuit connection is shown in Fig. 8.

Table 1. Function Table of 74HC4052

\begin{tabular}{lllc}
\hline Inputs & & & Channel On \\
\cline { 1 - 2 } Enable (INH) & $\mathrm{B}$ & $\mathrm{A}$ & \\
$\mathbf{0}$ & 0 & 0 & $\mathrm{X} 0, \mathrm{X}$ \\
$\mathbf{0}$ & 0 & 1 & $\mathrm{X} 1, \mathrm{X}$ \\
$\mathbf{0}$ & 1 & 0 & $\mathrm{Y} 0, \mathrm{Y}$ \\
$\mathbf{0}$ & 1 & 1 & $\mathrm{Y} 1, \mathrm{Y}$ \\
$\mathbf{1}$ & $\mathrm{x}$ & $\mathrm{x}$ & None \\
\hline
\end{tabular}

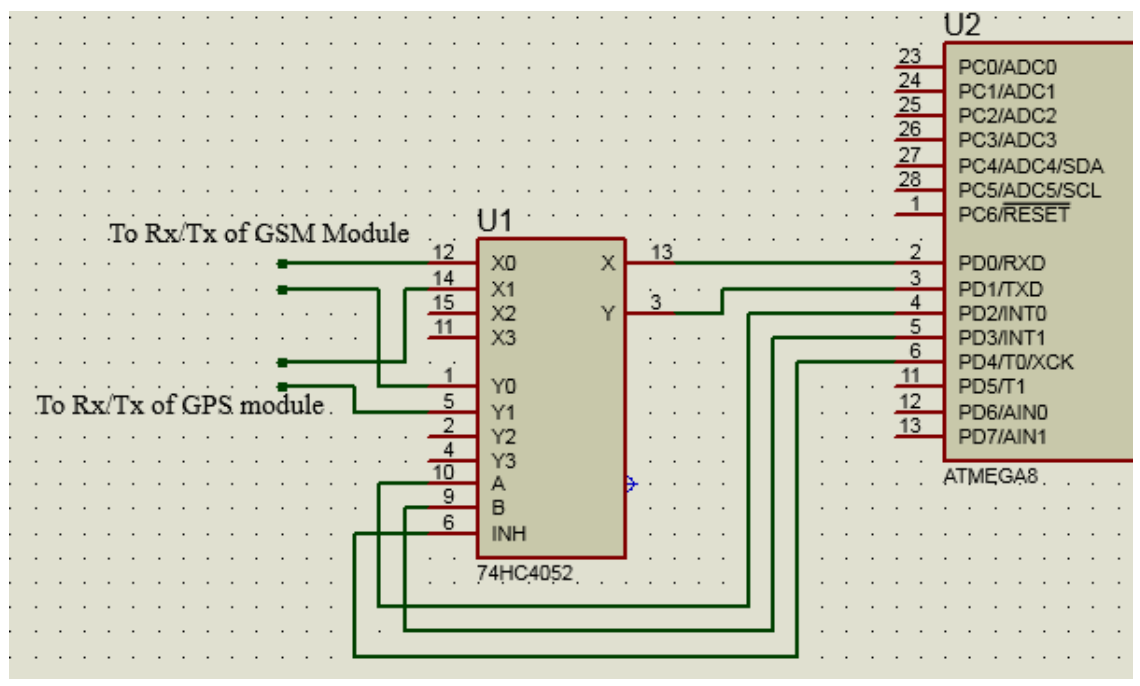

Fig.8. Serial Port Multiplexer (74HC4052) Connection to Atmega8 Microcontroller

Table 1 shows how the receiving port $(\mathrm{Rx})$ and transmitting port $(\mathrm{Tx})$ are selected using the selection pins $\mathrm{A}$ and $\mathrm{B}$. Pin $\mathrm{X}$ goes to receiving (RXD) pin of the microcontroller, while pin $\mathrm{Y}$ goes to the transmitting pin (TXD). X0 and Y0 are connected to the Tx and Rx pins of the GSM module respectively. Similarly X1 and Y1 for the GPS module. While enable, A and B are at logic low, the Tx pin of the GSM module is selected. However, when enable is high, no channel is active irrespective of the logic level at A and B. This is indicated by ' $x$ ' (don't care) as shown in Table 1 . In this way, the microcontroller is able to switch communication between the two modules.

\subsection{Software Design and Considerations}

The microcontroller controls the connected units using a software program code written using the Atmel studio 6.1. The pseudocode of the program is as shown in Fig. 9. The code was debugged and compiled. The generated Hex file was embedded into the microcontroller using Top 2013 universal IC programmer. 


\begin{tabular}{|l|l|}
\hline Start & \\
System initialization \\
Monitor pipeline surroundings \\
If motion detected \\
Send early notification \\
Monitor pipe sound and vibration \\
\\
If sound and vibration above threshold \\
\\
$\quad$ Send vandalism alert \\
End \\
Else $\quad$ Return \\
End
\end{tabular}

Fig.9. Program Pseudocode

On power-on, the system first initializes the sensors and the communication unit. The microcontroller reads the sensors to know the on-going activities around the pipeline. One important operation carried by the microcontroller is the conversion of sensors reading to digital version. To avoid false alarm, several samples were taken and the average value is obtained as:

$$
V_{s}=\frac{V_{s 1}+V_{s 2}+\ldots+V_{s n}}{n}
$$

Where $V_{s}$ is the average sound, and $V_{s n}$ are the n-samples read by the microcontroller from the sound sensor. The pipeline is considered to be under attack if:

$$
V_{s}>V_{t h}
$$

where $V_{t h}$ is the set threshold for sound detection. Similar analysis applies to the vibration sensor. The motion sensor module originally provides a digital output thus the above analysis is not needed for this sensor.

\section{Testing and Discussion of Results}

The setup used to test the proposed system is a desktop experimental setup as shown in Fig. 10. The sensors were placed around a test pipe of dimension: length $=565 \mathrm{~mm}$, outer diameter $=200 \mathrm{~mm}$, inner diameter $=160$ $\mathrm{mm}$ and pipe thickness $=2 \mathrm{~mm}$. 


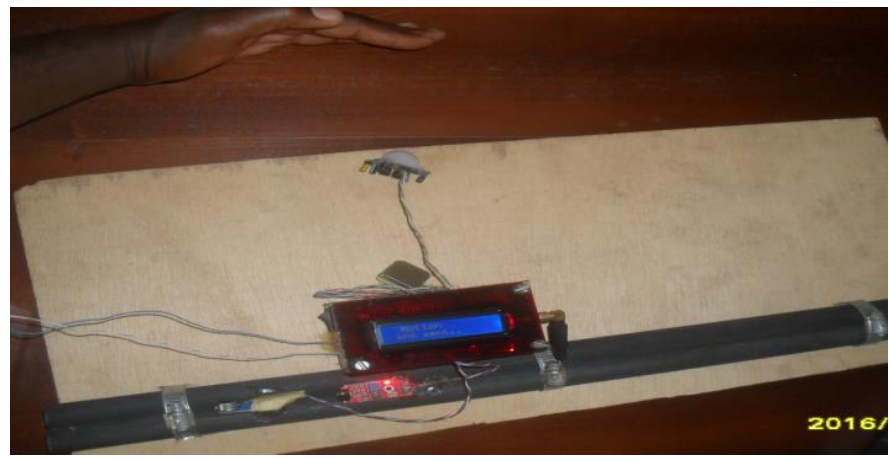

Fig.10. Experimental Setup during Motion Detection Testing

Fig. 11 shows a snapshot of the notification received when motion was detected around the setup. It contains the latitude and longitude of the pipeline location. This can help guide security personnel to reach the facilities without much stress.

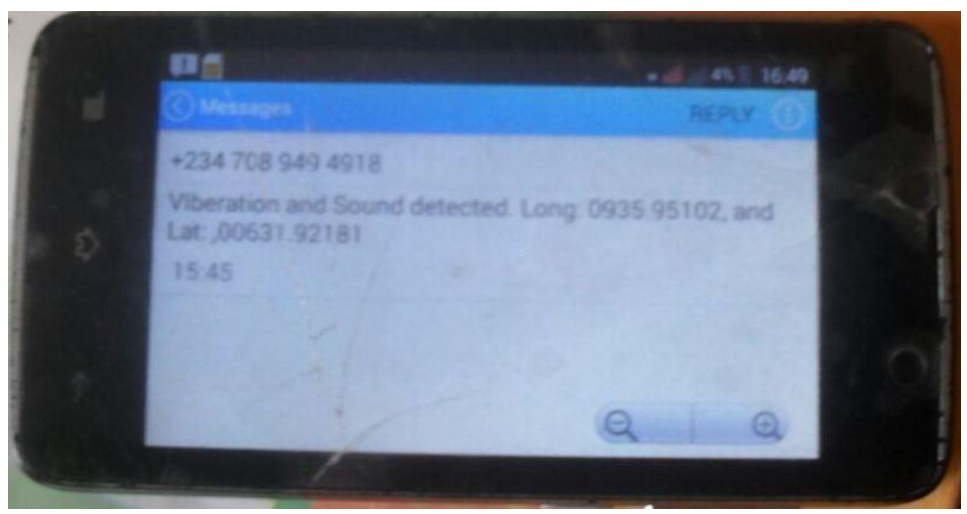

Fig.11. Snapshot Showing SMS Received When Motion Was Detected Around Setup.

Fig. 12 shows the setup for testing vibration and sound. This involved striking on or around the test pipe to evaluate the response of the sensors.

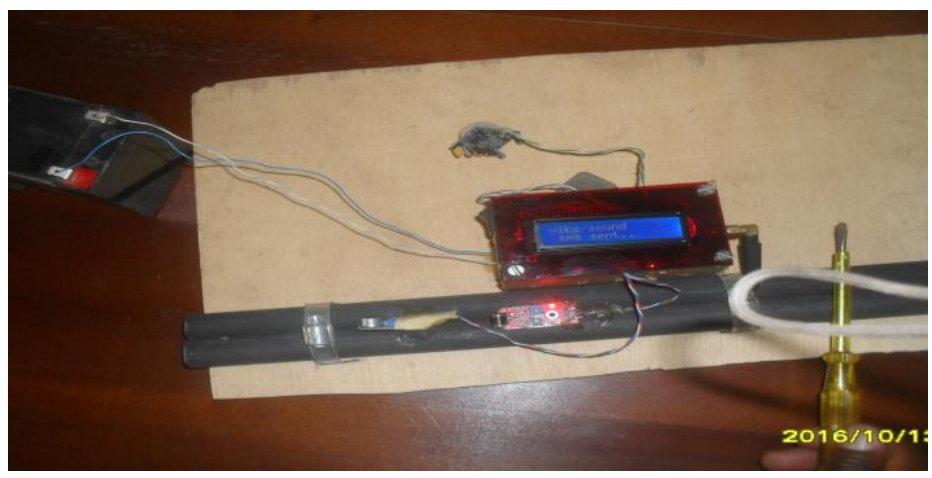

Fig.12. Snapshot Showing Setup used to Test Vibration and Sound Detection around the Pipeline. 
The sensor combination used in this design are such that the vandals can hardly noticed the presence of a monitoring device, unlike some previous systems where cameras were used. These cameras are first destroyed by vandals before they start their activities. The motion sensor plays a very important role when it comes to the issue of vandals noticing the presence of a monitoring device. Thus, the proposed device has the ability to still notify concern authorities of the presence of possible vandals before it can be destroyed by vandals in the event that vandals noticed the presence it. The overall system analysis of the proposed system suggests it as viable solution for monitoring pipeline system with ease.

\section{Conclusion}

In this paper, a multi-sensor approach for pipeline monitoring was presented. The system is capable of detecting movement of possible vandals around pipeline facilities and pre-informing concern personnel. In the event of detecting vandalism activities, a second notification is sent including the GPS coordinates of the location. Future works will include an adaptive communication method such that the control unit selects the network with the strongest signal strength in sending the alert to concern authorities, an adaptive thresholding method to determine the nature of the sound picked by the sound sensor.

\section{References}

[1] PM News, "Nigeria loses N470m daily to pipeline vandalism," 2016. [Online]. Available: http://www.news24.com.ng/National/News/nigeria-loses-n470m-daily-to-pipeline-vandalism-20160120. [Accessed: 24-Apr-2016].

[2] O. Shoewu, L. A. Akinyemi, K. A. Ayanlowo, S. O. Olatinwo, and N. T. Makanjuola, "Development of a Microcontroller Based Alarm System for Pipeline Vandals Detection," J. Sci. Eng., vol. 1, pp. 133$142,2013$.

[3] F. C. Obodoeze, "Wireless Sensor Network in Niger Delta Oil and Gas Field Monitoring: The Security Challenges and Countermeasures," Int. J. Distrib. Parallel Syst., vol. 3, no. 6, pp. 65-77, 2012.

[4] I. Abraham, "Designing an Intelligent Microcontroller based Pipeline Monitoring System with Alarm , Sensor," vol. 3, no. 2, pp. 22-27, 2015.

[5] V. O. S. Olunloyo, A. M. Ajofoyinbo, and A. Yaba, "A model for real time leakage detection in pipelines : A case of an integrated GPS receiver."

[6] K. F. K. Oyedeko and P. Engineering, "Modeling and Simulation of a Leak Detection for Oil and Gas Pipelines via Transient Model : A Case Study of the Niger Delta," vol. 5, no. 1, pp. 16-28, 2015.

[7] Z. Yang, "Research on Leakage Detection and Analysis of Leakage Point in the Gas Pipeline System," Open J. Saf. Sci. Technol., vol. 1, no. 3, pp. 94-100, 2011.

[8] G. N. Ezeh, N. Chukwuchekwa, J. C. Ojiaku, and E. Ekeanyawu, "Pipeline Vandalisation Detection Alert with Sms," vol. 4, no. 4, pp. 21-25, 2014.

[9] T. Chis and A. Saguna, "Pipeline Leak Detection Techniques," Ann. Comput. Sci. Ser. 5th Tome 1st Fasc., pp. 25-34.

[10] N. F. Henry and O. N. Henry, "Wireless Sensor Networks based Pipeline Vandalisation and Oil Spillage Monitoring and Detection: Main Benefits for Nigeria Oil and Gas Sectors," vol. 3, no. 1, pp. 1-7, 2015.

[11] A. Azubogu, V. Idigo, S. Nnebe, and O. Oguejiofor, "Wireless Sensor Networks for Long Distance Pipeline Monitoring," Waset.Org, vol. 7, no. 3, pp. 86-91, 2013.

[12] H. Zhang, F. Zhang, Y. Wang, and G. Zhang, "Wireless Sensor Network based anti-theft system of monitoring on petroleum pipeline," 2011 2nd Int. Conf. Mech. Autom. Control Eng. MACE 2011 - Proc., pp. 4689-4692, 2011.

[13] Z. Chen, M. Temitayo, and P. D. Isa, "Pipe Flaws Detection by Using the Mindstorm Robot," vol. 2, no. 6, pp. 569-574, 2012. 
[14] G. C. Ononiwu, P. U. Eze, O. J. Onojo, G. N. Ezeh, D. O. Dike, S. I. Igbojiaku, and O. C. Nnodi, “A Real-Time Oil Pipeline Anti-Intrusion System Using Acoustic Sensors," vol. 4, no. 26, pp. 3740-3756, 2014.

[15] S. Choi, B. Song, R. Ha, and H. Cha, "Energy-aware pipeline monitoring system using piezoelectric sensor," IEEE Sens. J., vol. 12, no. 6, pp. 1695-1702, 2012.

[16] N. Mohamed and I. Jawhar, "A fault tolerant wired/wireless sensor network architecture for monitoring pipeline infrastructures," Proc. - 2nd Int. Conf. Sens. Technol. Appl., SENSORCOMM 2008, Incl. MESH 2008 Conf. Mesh Networks; ENOPT 2008 Energy Optim. Wirel. Sensors Networks, UNWAT 2008 Under Water Sensors Syst., pp. 179-184, 2008.

[17] L. Qiu, K. I. Wang, and Z. Salcic, "Dynamic Duty Cycle-based Wireless Sensor Network for Underground Pipeline Monitoring," pp. 116-121, 2015.

[18] H. Saeed, S. Ali, S. Rashid, S. Qaisar, and E. Felemban, "Reliable monitoring of oil and gas pipelines using wireless sensor network (WSN) - REMONG," 2014 9th Int. Conf. Syst. Syst. Eng., pp. 230-235, 2014.

[19] R. B. Santos, E. O. De Sousa, F. V. Da Silva, S. L. Da Cruz, and a. M. F. Fileti, "Real-Time Monitoring of Gas Pipeline through Artificial Neural Networks," 2013 BRICS Congr. Comput. Intell. 11th Brazilian Congr. Comput. Intell., pp. 329-334, 2013.

[20] J. Sun and J. Wen, "Research on monitoring and pre-warning system for security of pipelines based on multi-seismic sensors," ICEMI 2009 - Proc. 9th Int. Conf. Electron. Meas. Instruments, pp. 1689-1693, 2009.

[21] H. Yan, G. Shi, S. Hao, and Q. Wang, "Oil Pipeline Safety Monitoring Method based on Vibration Signal Analysis and Recognition,” 2009 WRI Glob. Congr. Intell. Syst., pp. 200-206, 2009.

[22] H. Yu and M. Guo, "An efficient oil and gas pipeline monitoring systems based on wireless sensor networks,” Inf. Secur. Intell. Control (ISIC), 2012 Int. Conf., pp. 178-181, 2012.

\section{Authors' Profiles}

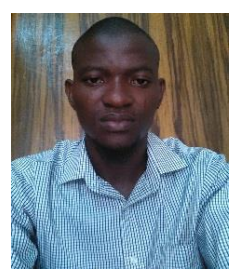

Smart Grid.

Salihu O. Aliyu is currently an Assistant Lecturer at the Department of Telecommunication Engineering, Federal University of Technology, Minna, Niger State Nigeria. He obtained a First Class honor, BEng. Electrical and Computer Engineering at Federal University of Technology, Minna, Nigeria in 2011, after which he proceed to King Fahd University of Petroleum and Minerals, Dhahran, Saudi Arabia where he obtained his MSc in Electrical Engineering. His research is focused on Digital Signal/Image processing, Embedded System Design, Artificial Intelligence, Pattern Recognition using machine learning algorithm and

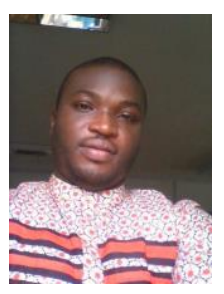

Ojela Innocent Agbo had his Bachelor of Engineering in Telecommunication Engineering from the Department of Telecommunication Engineering, Federal University of Technology, Minna, Niger State Nigeria in 2016. He is a promising embedded system specialist. His areas of research includes embedded, and Intelligent Systems design. 


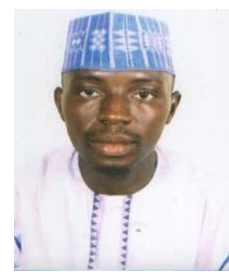

Mr. Saidu Muslim obtained a Bachelor of Engineering (B.Eng.) Degree from Electrical and Computer Engineering Department, Federal University of Technology (FUT) Minna, Niger State, Nigeria, in December, 2008 and a Master of Engineering (M.Eng.) Degree, in Telecommunication Engineering from Telecommunication Engineering Department, FUT, Minna, Niger State, Nigeria, in June 2014. He is currently an Assistant Lecturer in the Department of Telecommunication Engineering, Federal University of Technology, Minna Nigeria. His Research interests includes Wireless Communication and Energy Resource Management in Wireless Sensor Network.

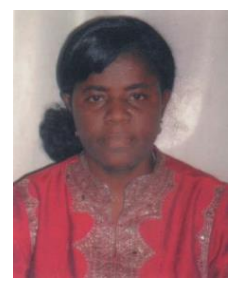

Elizabeth N. Onwuka obtained a Bachelor of Engineering (B.Eng.) Degree from Electrical and Computer Engineering Department, Federal University of Technology (FUT) Minna, Niger State, Nigeria, in October 1992; a Master of Engineering (M.Eng.) Degree, in Telecommunications, from Electrical and Computer Engineering Department, FUT, Minna, Niger State, Nigeria, in March 1998; and Doctor of Philosophy (PhD) Degree, in Communications and Information Systems Engineering, from Tsinghua University, Beijing, People's Republic of China, in June 2004. She is currently a Professor in the Department of Telecommunications Engineering FUT, Minna, Niger state. Her research interest includes Mobile communications network architecture, IP networks, handoff management, paging, network integration, and resource management in wireless networks, wireless sensor networks.

How to cite this paper: Salihu O. Aliyu, Innocent O. Agbo, Saidu Muslim, Elizabeth N. Onwuka,"MultiSensor Approach for Monitoring Pipelines", International Journal of Engineering and Manufacturing(IJEM), Vol.7, No.6, pp.59-72, 2017.DOI: 10.5815/ijem.2017.06.06 